\title{
Ginástica hipopressiva: as evidências de uma alternativa ao treinamento da musculatura do assoalho pélvico de mulheres com déficit proprioceptivo local Hipopressive gymnastics: evidences for an alternative training for women with local proprioceptive deficit of the pelvic floor muscles
}

Gustavo Fernando Sutter Latorre, Ft.*, Maura Regina Seleme, Ft., D.Sc.**, Ana Paula Magalhães Resende, Ft., D.Sc.***, Bary Berghmans, Ft., D.Sc.****

* Mestrando do Programa de Pós-Graduação em Fisioterapia, Universidade do Estado de Santa Catarina - UDESC,

**Doutora pela Universidade Federal do Rio de Janeiro - UFRJ, ${ }^{* * *}$ Doutora pela Universidade Federal de São Paulo - UNIFESP,

***PhD em Epidemiologia Clínica, Fisioterapeuta Especialista no Assoalho Pélvico, Universidade de Maastricht, Holanda

\section{Resumo}

Apesar de a cinesioterapia para o assoalho pélvico fazer parte do padrão áureo para o tratamento das disfunçôes do assoalho pélvico, um terço das mulheres apresenta dificuldade de identificação daquela musculatura o que tolhe o início do tratamento. A ginástica hipopressiva é técnica recente que promete contração reflexa da musculatura do assoalho pélvico, mas é parca a evidência a este respeito. Métodos: Organização da evidência, especialmente por exames de imagem e eletromiografia, publicada até o momento. Resultados: A ginástica hipopressiva provoca momento cranial das vísceras, o que pode ser observado por ultrassonografia, ressonância magnética e observaçáo direta via espéculo. A eletromiografia durante a manobra sugere ativação reflexa da musculatura do assoalho pélvico. Conclusão: A ginástica hipopressiva age no assoalho pélvico, e pode ser utilizada como técnica adjuvante para o despertar proprioceptivo da musculatura local.

Palavras-chave: ginástica hipopressiva, cinesioterapia, assoalho pélvico, propriocepção, fisioterapia.

\section{Introdução}

Completamos, desde os estudos pioneiros de Kegel, mais de 60 anos de sucesso no exercício da musculatura do assoalho pélvico (MAP) no tratamento das disfunçóes do assoalho pélvico - dentre as quais se destacam as incontinências urinária e fecal, os prolapsos genitais e disfunções sexuais diversas, sucesso este corroborado por revisões sistemáticas

\begin{abstract}
Although pelvic floor kinesiotherapy is, today, part of the gold standard for the treatment of pelvic floor dysfunction, one-third of women have local proprioceptive difficulty, what hinders the initiation of treatment. The hipopressive gymnastic is a new technique that promises reflex contraction of the pelvic floor muscles, but the evidence is sparse. Methods: Organizing the evidence, especially for imaging and electromyography, published so far. Results: Hipopressive gymnastics causes cranial momentum of the pelvic organs, which can be seen by ultrasound, magnetic resonance and direct observation by speculum. Electromyography suggests reflex activation of the pelvic floor muscles. Conclusion: Hipopressive gymnastics acts on the pelvic floor, and can be used as an adjuvant technique for the proprioceptive awakening of pelvic floor muscle.
\end{abstract}

Key-words: hipopressive gymnastic, kinesiotherapy, pelvic floor, proprioception, physiotherapy.

recentes [1] e, ao ponto de, hoje, a fisioterapia uroginecológica ser considerada parte indispensável no tratamento de padrão áureo, recomendado pela International Continence Society [2]. Das ferramentas utilizadas pelo fisioterapeuta especializado tem destaque a cinesioterapia, cuja eficácia têm se evidenciado superior à eletroterapia e outros tipos alternativos de tratamento quando comparada em estudos randômicos controlados [3]. 
Um dos maiores desafios clínicos no tratamento cinesioterápico da mulher com disfunção do assoalho pélvico é o despertar proprioceptivo de uma região que, especialmente na cultura ocidental, é associado à baixa consciência corporal. A MAP não é facilmente reconhecida pelas mulheres [4], e mais de 30\% das mulheres é incapaz de contrair adequadamente aquela musculatura [5]. É neste âmbito que novas técnicas cinesioterápicas que, de alguma forma, possam despertar a propriocepção local de um modo distinto da abordagem invasiva tradicional, podem vir a fazer diferença. Uma destas técnicas, a Ginástica Hipopressiva $(\mathrm{GH})$, vem lentamente conquistando espaço nos tratamentos uroginecológicos.

Técnica recente, criada por pesquisadores franceses na década de 1980, a GH, baseada na contração ativa dos abdominais - manobra de aspiração diafragmática - e respectiva contração, supostamente reflexa da MAP, apesar de utilizada por profissionais e ser parte de cursos de capacitação em nosso país, ainda carece de evidências científicas concretas. Organizar a evidência visual disponível hoje é, por fim, a razão do presente estudo.

\section{Material e métodos}

Trata-se da organização dos dados de um esforço multicêntrico no qual foi efetuada inicialmente uma busca pelas publicaçóes originais na Europa para os princípios da GH. Para evidenciar a ação da manobra de aspiração diafragmática - o exercício principal da GH - sobre as estruturas pélvicas femininas o exercício foi registrado de diferentes formas: 1 ) por filmagem em vídeo sob visão especular da região urogenital feminina e dos órgãos pélvicos durante a manobra; 2) por mensuração da mudança no ângulo entre uretra $e$ parede vaginal através de imagem ultrassonográfica; 3) pela mensuração da distância entre os elevadores do ânus e o sacro através de imagens de ressonância magnética; 4) mensuração da mudança no ângulo entre útero e canal vaginal através de imagens de ressonância magnética; 5) da mensuração das atividades eletromiográficas da musculatura abdominal, ativa, e da MAP, supostamente reflexa, simultaneamente durante a manobra; 6) mensuração através de sistema de biofeedback da atividade da musculatura abdominal durante a manobra, sem contração ativa da MAP em decúbito dorsal; 7) mensuração através de sistema de biofeedback da atividade isolada da MAP durante a manobra, sem contração ativa abdominal em decúbito dorsal; 8) mensuração através de sistema de biofeedback da atividade da MAP simultaneamente à manobra de aspiração diafragmática em decúbito dorsal; 9) mensuração por biofeedback da atividade abdominal durante a manobra, sem contração ativa da MAP em decúbito ventral; 7) mensuração por biofeedback da atividade isolada da MAP durante a manobra, sem contraçáo ativa abdominal em decúbito ventral; 8) mensuração através de sistema de biofeedback da atividade da MAP simultaneamente à manobra de aspiração diafragmática em decúbito ventral.

\section{Resultados}

A tese de Marcel Caufriez, 1991, descrevia a GH em quatro movimentos: inspiração diafragmática lenta e profunda; seguida de expiração lenta e profunda; seguida de uma contração progressiva dos músculos transversos abdominais aliados aos intercostais, que ele chamou de "aspiração diafragmática", terminada numa apnéia de 10 segundos.

$\mathrm{O}$ vídeo da visualização interna do canal vaginal sob especulo, realizado na Espanha por J. Amostegui [6], mostra o comportamento da cavidade vaginal profunda durante a realização da manobra de aspiração diafragmática. É possível visualizar claramente a elevação da parede vaginal anterior e, especialmente, do fundo de saco uterino, evidenciando diretamente a criação de momento cinético caudo-cranial do útero e da parede vaginal anterior.

\section{Figura 1 - Ultrassonografia do ângulo entre uretra e parede vesical durante a manobra de aspiraçâo diafragmática.}

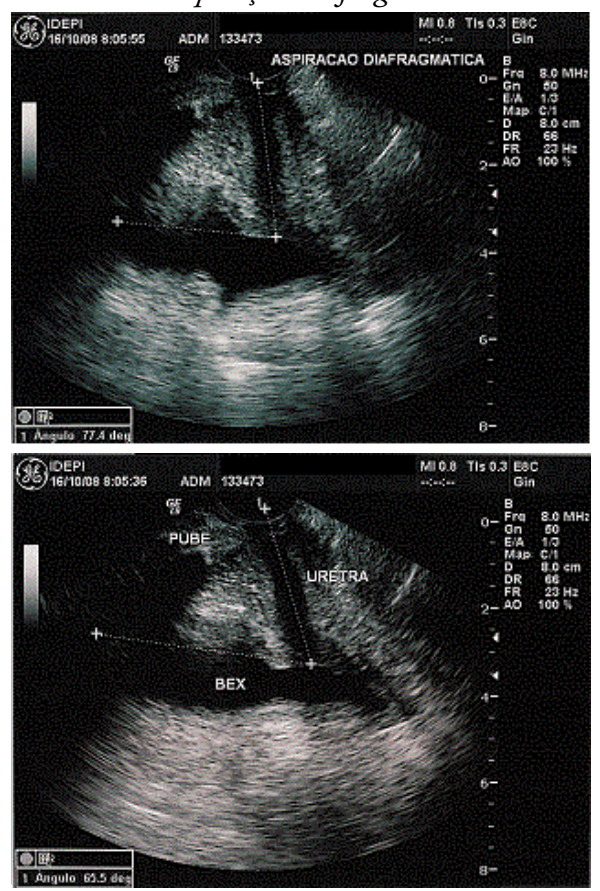

ângulo entre a uretra e a parede vaginal aumenta $12^{\circ}$ a partir dos $65^{\circ}$ durante o repouso, para $77^{\circ}$ durante a manobra de aspiração diafragmática [7].

A sequência de imagens (Figura 2) ultrassonográficas mostrou que o ângulo entre a uretra e a parede vaginal aumentou em $12^{\circ}$ (de $65^{\circ}$ para $77^{\circ}$ ) durante a manobra de aspiração diafragmática [7]. A distância entre a MAP e o promontório sacral, em imagens de ressonância magnética, é diminuída de $83,8 \mathrm{~mm}$ para 76,8 $\mathrm{mm}$, evidenciando novamente momento caudo-cranial [7]. O aumento no ângulo agudo formado pelo útero anterovertido com o canal vaginal, de $31^{\circ}$ para $41^{\circ}$, visualizado por ressonância magnética, durante a manobra, comprova o momento caudo-cranial causado por aquela sobre aquele órgão [7]. 
Figura 2 - Imagens de ressonância magnética mostrando o comportamento da musculatura abdominal, útero e vagina durante a manobra de aspiração diafragmática.

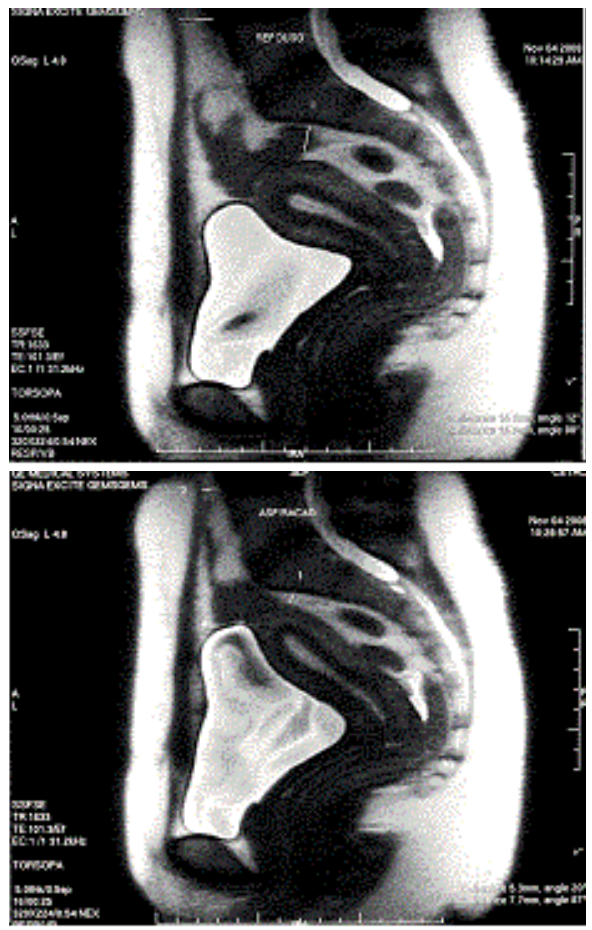

A primeira imagem mostra os músculos abdominais durante o repouso, e a segunda durante a manobra de aspiração diafragmática. $\bigcirc$ ângulo entre útero e vagina muda de $37^{\circ} \mathrm{em}$ repouso (cima) para $45^{\circ}$ graus após a manobra (baixo).
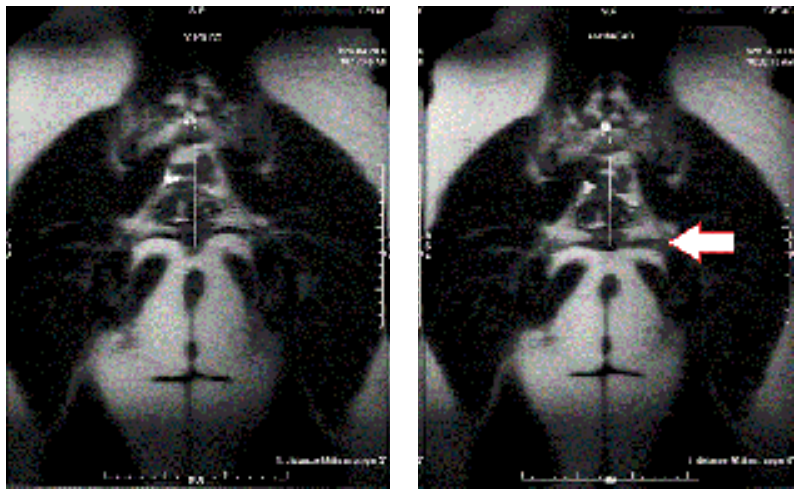

Estas imagens mostram a alteração na distância entre os elevadores do ânus e o sacro, de 83,8 milímetros durante o repouso (esquerda) para $76,8 \mathrm{~mm}$ durante uma aspiração diafragmática (direita) [7].

O estudo eletromiográfico mostra a atividade da musculatura abdominal e alguma atividade da MAP, que acontece milésimos de segundo depois da primeira, em uma mulher treinada que, em tese, saberia manter a MAP relaxada enquanto realizava a manobra de aspiração, sugerindo que contração abdominal utilizada naquela manobra realizaria uma ativação reflexa da MAP [8].

Através do sistema de biofeedback intracavitário também foi possível observar que durante a manobra de aspiração dia- fragmática há alguma atividade da MAP. Ainda, a contração da MAP quando realizada em conjunto com a manobra de aspiraçáo diafragmática mostrou-se visivelmente maior do que quando realizada de maneira isolada. Este efeito é verificado tanto quando a manobra é efetuada na posição em decúbito dorsal, quanto naquela realizada em decúbito ventral [9].

\section{Discussão}

A parede vaginal anterior é intimamente relacionada à bexiga [10] e a manobra de aspiração diafragmática causa momento caudo-cranial na parede vaginal anterior. Evidencia-se indiretamente o momento caudo-cranial uretral por conta da manobra de aspiração. De fato, a uretra é movida cranialmente durante a manobra, conforme mostrado pela sequência de imagens ultrassonográficas em planos distintos e, não só ela, como também a bexiga e o útero são elevados.

Caufriez [11] descrevia que a manobra de aspiração diafragmática provoca a elevação dos órgãos pélvicos na direção do diafragma respiratório, induzindo uma contração reflexa da MAP. Exatamente o mesmo foi sugerido pelo estudo eletromiográfico realizado [8] e pelo estudo através de biofeedback [9]. No entanto, mais estudos são necessários para o esclarecimento sólido a este respeito, especialmente com um número maior de mulheres participantes. Foi demonstrado, ainda, que a ginástica hipopressiva produz seus efeitos quando realizada tanto na posição em decúbito ventral quanto em decúbito dorsal [9], facilitando o acesso a pacientes acamados que permitam tratamento em apenas um plano corporal.

Em 1991 a tese de doutorado inicial de Marcel Caufriez descrevia a GH como uma técnica adjuvante, e não o tratamento em si. Bertotto \& Costa [12] descreveram significativa atividade reflexa da MAP verificada eletromiograficamente durante a realização da GH. Bertotto \& Rodrigues [13] descreveram em estudo piloto clínico náo controlado que o grupo submetido aos exercícios de contração simples da MAP tiveram resultado inferior ao grupo submetido aos mesmos exercícios associados à GH.

Recentemente foi realizado um estudo com nulíparas que avaliou o comportamento do diafragma e da MAP durante a respiraçáo tranqüila por meio de ressonância magnética. Foi observado que, durante a inspiração, quando os pulmôes se enchiam havia o rebaixamento das cúpulas diafragmáticas. A MAP apresentou comportamento semelhante, de descida, acompanhando o momento do diafragma durante a respiração. Por outro lado, durante a expiração, tanto as cúpulas diafragmáticas quanto a MAP se elevaram, sugerindo sinergia entre estes músculos durante aquele movimento [14]. Estes achados estão em acordo com a teoria proposta pela $\mathrm{GH}$, que afirma haver elevação das cúpulas diafragmáticas durante as manobras de aspiração diafragmática, de modo concomitante à elevação reflexa dos órgãos pélvicos e do assoalho pélvico e si [15].

Em estudo randomizado controlado comparando dados eletromiográficos das atividades dos transversos abdominais 
e da MAP durante a contração ativa dos elevadores do ânus e a manobra de aspiração diafragmática em 34 nulíparas, Stüpp et al. [16] mostraram que a manobra de aspiraçáo, de fato, produz ativação da MAP quando comparado ao seu estado de repouso. Esta ativação, como esperado, foi menor do que a ativação da MAP gerada pela contração ativa daquela musculatura. No entanto, quando a contraçáo dos elevadores foi realizada simultaneamente à manobra de aspiração diafragmática, a ativaçáo do assoalho pélvico não foi significativamente maior do que a contraçáo isolada da MAP, sugerindo que não haveria indicação para a utilizaçáo da GH quando objetivo for potencializar a contração dos elevadores, mas que, em virtude da ativaçáo involuntária da MAP durante a manobra de aspiraçáo, a GH pode ser útil como adjuvante para os casos onde a mulher é, por algum motivo, incapaz de contrair conscientemente a musculatura do seu assoalho pélvico.

Por incitar a atividade reflexa daquela musculatura, a $\mathrm{GH}$ pode ser uma alternativa interessante de tratamento ou, ao menos, ser um ponto de partida para que a conscientizaçáo da MAP possa ser despertada e, finalmente, para que os exercícios de contração direta possam ser realizados com eficácia.

\section{Conclusão}

A GH promove a elevação das vísceras pélvicas, ao causar um momento cinético cranial verificável através de observação por especulo, ressonância magnética e ultrassonografia. Suas manobras causam uma ativação da MAP aparentemente reflexa, verificada em estudos eletromiográficos de superfície e por biofeedback intracavitário. Quando realizada concomitantemente à $\mathrm{GH}$ a contração da MAP é estimulada, mas não potencializada. Os resultados independem de a manobra ser realizada com a mulher em decúbito ventral ou dorsal. Como técnica a ser adicionada ao arsenal fisioterápico, a GH pode ser útil para mulheres com dificuldade proprioceptiva para quanto ao seu assoalho pélvico, tanto para permitir um início de propriocepção que permita o reconhecimento da área para posterior exercício de contração da MAP, quanto para potencializar a contração da MAP durante seu exercício; nos casos do pós-parto imediato quando a contração da MAP é ineficiente e em todos os casos onde a contração da MAP é insuficiente, ineficaz, ou impossibilitada por um motivo qualquer.

\section{Referências}

1. Price N, Dawood R, Jackson SR. Pelvic floor exercise for urinary incontinence: a systematic literature review. Maturitas 2010 Dec;67(4):309-15.

2. Berghmans $\mathrm{B}, \mathrm{B} ø \mathrm{~K}$, Bernards $\mathrm{N}$ et al. Clinical practice guidelines for the physical therapy of patients with stress urinary incontinence. Urodinâmica e uroginecologia 2003;6(1):1-14.

3. Bø k, Talseth T, Holme I. Single blind, randomised controlled trial of pelvis floor exercises, eletrical stimulation, vaginal cones, and no treatment in management of genuine stress incontinence in women. BMJ 1999;318:487-93.

4. Bump RC, Hurt WG, Fantl JA, Wyman JF. Assessment of Kegel pelvic muscle exercise performance after brief verbal instruction. Am J Obstet Gynecol 1991, 165(2):322-7;327-9.

5. $\mathrm{B} ø \mathrm{~K}$ et al. Evidence based physiotherapy for the pelvic floor. Bridging science and clinical practice. Elsevier; 2007.

6. Seleme MR, Dabadie L. The hypopressive gymnastic. 36 Annual Meeting of the International Urogynecological Association - IUGA 2011, Lisboa, Portugal.

7. Berghmans B et al. Pelvic floor reabilitation. In: David Staskin DR, ed. Atlas of bladder disease. Berlin: Springer; 2010.

8. Seleme MR, Dabbadie L, Ramos L. Experimento eletromiográfico da manobra de aspiração diafragmática. Realizado na Escola Superior de Tecnologia da Saúde de Lisboa. Portugal: Research Labs; 2008.

9. Seleme MR. Ginastica hipopressiva in urofisioterapia - aplicaçôes técnicas fisioterapeuticas nas disfunçoes miccionais e do assoalho pélvico. Sáo Paulo: Paulo Palma; 2009.

10. Ashton-Miller JA, DeLancey JOL. Functional anatomy of the female pelvic floor. In: Annals of The New York Academy of Sciences. Special Volume on Reproductive Biomechanics; 2007.

11. Caufriez M. Gymnastique abdominale hypopressive. Bruxelles; 1997. p.8-10.

12. Bertotto A, Costa T. Avaliação do assoalho pélvico em mulheres adultas submetidas à técnica abdominal hipopressiva. [TCC] Canoas: Universidade Unilasalle, Curso de Fisioterapia; 2008.

13. Bertotto A, Rodriguez B. Treinamento dos músculos perineais versus técnica abdominal hipopressiva: tratamento preventivo e conservador das disfunçóes do assoalho pélvico avaliados através do biofeedback eletromiográfico. [TCC]. Canoas: Universidade Unilasalle, Curso de Fisioterapia; 2008.

14. Talasz H, Kremser C, Kofler M, Kalchschmid E, Lechleitner M, Rudisch A. Phase-locked parallel movement of diaphragm and pelvic floor during breathing and coughing - a dynamic MRI investigation in healthy females. Int Urogynecol J 2011;22:61-8.

15. Seleme MR, Bertotto A, Ribeiro VW. Exercícios hipopressivos In: Palma P, ed. Urofisioterapia: Aplicaçôes clínicas das técnicas fisioterapêuticas nas disfunçôes miccionais e do assoalho pélvico. Personal link; 2009. p.295-307.

16. Stüpp L, Resende APM, Petricelli, CD et al. Pelvic floor muscle and transverses abdominis activation in abdominal hypopressive technique through surface electromyography. Neurourol Urodyn 2011;30(8):1518-21. 